\title{
A Deep Learning Approach for Safety Monitoring of Sick People
}

\author{
GP Ramesh ${ }^{1 *}$ and Hemalatha K.L. ${ }^{2}$ \\ ${ }^{1}$ Department of Electronics \& Communication Engineering, St. Peters Institute of Higher Education and \\ Research, Avadi, Tamil Nadu, India \\ ${ }^{2}$ Department of Information Science \& Engineering Sri Krishna Institute of Technology, Bangalore, India
}

\begin{abstract}
This project illustrates a method of smart recognition of human behavior to automatically recognize human actions from skeletal joint movements and integrate the skills. This is a low-cost solution and has high precision. An independent mobile app is also intended to track the condition of individuals and their environment while they are alone. The mobile application also incorporates a Notification API integration to enable the sending of warning alerts during irregular conditions. Therefore, our initiative offers a way to assist senior citizens and children with some kind of mishap and health problems. This research proposed novel Convolutional Neural Network (ConvNet/CNN) to predict the action based on human activity. Proposed model provides 3\% better accuracy than the existing state of art methodologies.
\end{abstract}

KEY WORDS: ABNORMAL ACTIVITY, ACTION RECOGNITION, AUGMENTED DATA, CONVOLUTIONAL NEURAL NETWORK, LOW COST

\section{INTRODUCTION}

The aim of the project is to classify human behaviors using skeletal joint movements. To create an intelligent system of human behavior and recognition of human movements that can automatically recognize human daily activities using human skeleton data, integrating image processing and deep learning techniques. Here we are going to capture the different skeletal images of human actions and collect it as data sets, train the data based on algorithms using deep learning. We will develop a react native application where we can view the live streaming of our house when an abnormal activity is detected. In this project, we are going to determine the human activity recognition by which we can help the elder ones as well

\section{ARTICLE INFORMATION}

*Corresponding Author: ; rameshgp@yahoo.com

Received 11th Oct 2020 Accepted after revision 29th Dec 2020

Print ISSN: 0974-6455 Online ISSN: 2321-4007 CODEN: BBRCBA

Thomson Reuters ISI Web of Science Clarivate Analytics USA and Crossref Indexed Journal

\section{Clarivate
Analytics}

NAAS Journal Score 2020 (4.31)

A Society of Science and Nature Publication,

Bhopal India 2020. All rights reserved.

Online Contents Available at: http//www.bbrc.in/

Doi: http://dx.doi.org/10.21786/bbrc/13.13/55 as children's living in home alone. So, the first step in the project will be collecting the dataset from various resources and then we will be separating these datasets into training as well as testing dataset where the testing dataset will be kept separate and the training dataset will be used to train the model. Then dataset augmentation will be done which will increase the dataset into multiple numbers. Then these datasets are pre-processed using different techniques to align the datasets into single dimensions. After pre-processing our dataset we will be ready for training with the architecture. Now, we will be using architecture such as VGG16 to train the model.

This paper is organized as follows: Section 2 provides a brief description of related works. Section 3 discussed on CNN method. Section 4 discussed results and discussion and Section 5 made the conclusion of this paper.

2. Related Works: Due to the presence of the pose and temporal changes in the action images, Suraj Prakash Sahoo et.al have suggested that identification of human action (HAR) is a difficult activity. In this paper, HARDepth is proposed with sequential and shape learning along with the new definition of depth history picture to overcome these difficulties (DHI). The proposed

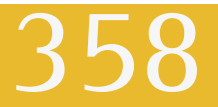


work is tested on publicly accessible datasets such as KTH, UCF sports, JHMDB, UCF101, and HMDB51 and achieves 97.67\%, 95.00\%, 73.13\%, 92.97\%, and 69.74\% performance accuracy, respectively. The findings on these datasets suggest that in terms of overall precision, kappa parameter and accuracy, the proposed work of this paper performs better compared to the other state-ofthe-art algorithms present in the previously published literature.

Figure 1: System Architecture

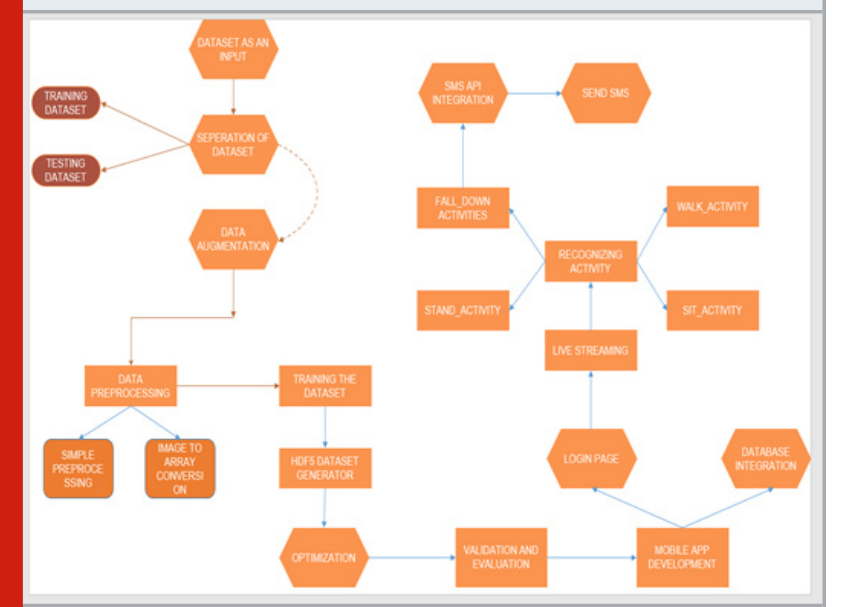

Figure 2: Traditional Convolutional Neural Network

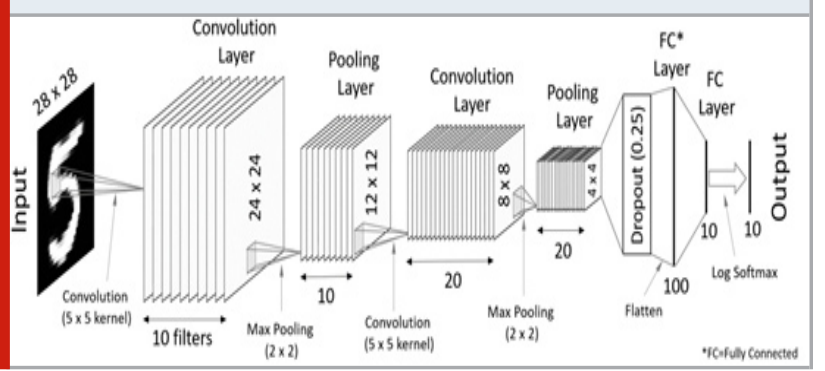

3. Proposed Method: CNN (Convolutional Neural Network) is a multi-layered neural network with a special architecture designed to evaluate the output by extracting increasingly complex data characteristics from each layer. For perceptual activities, CNNs are well adapted.

The CNN receives a picture of a cat, let's say, this image is a pixel set in computer terms. The network can recognise specific features during feature learning (i.e. hidden layers), such as the cat's tail, ear, etc. As research continues in applications such as tracking the elderly and surveillance for the detection of suspicious people and items left in public places, the potential of machine learning, and particularly deep learning, has become evident. Although some techniques have been developed for Human Action Recognition (HAR) using these devices, people, particularly children and the elderly, can experience excessive mental and physical discomfort. Therefore, research has centered on imagebased HAR, positioning it on the front line of innovations in consumer electronics.
This paper proposes an intelligent system of human action detection that can automatically identify human everyday activities using human skeleton knowledge from depth sensors, incorporating image processing and deep learning techniques. In addition, an approach using skeleton knowledge has proven very promising due to low computational costs and high accuracy performance, and can be used without any constraints on environments or domain structures. This paper therefore explores the creation of an efficient HAR based on skeleton data that can be used as an embedded framework. Two popular public data sets of human everyday activities are used to conduct the experiments. The suggested system outperforms other state-of-the-art approaches on both datasets, according to the experimental results.

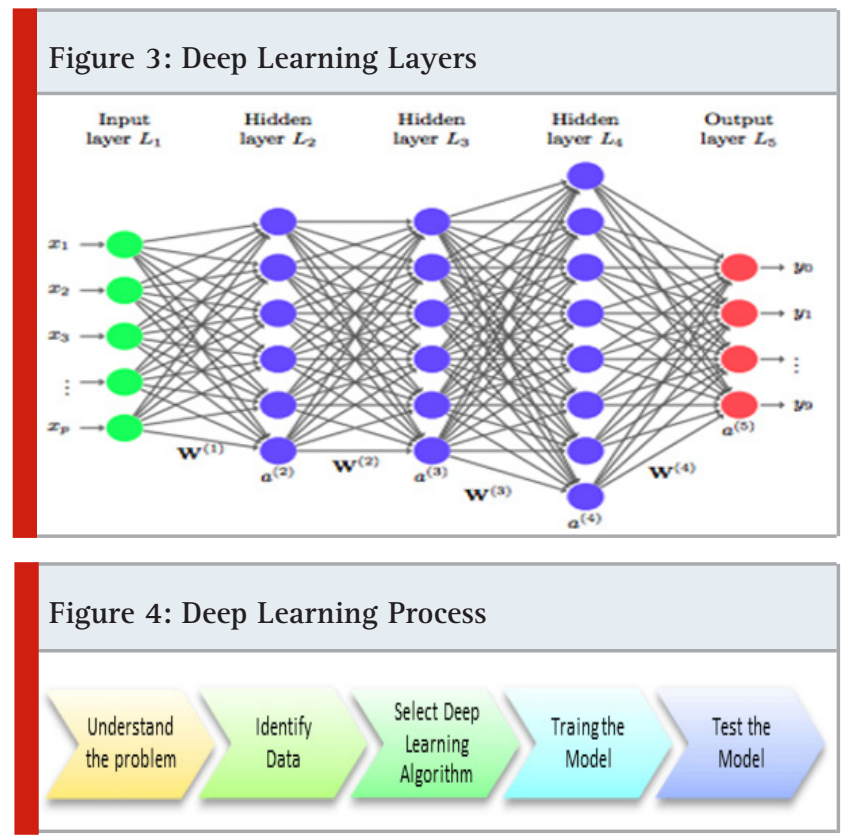

\begin{tabular}{|c|c|}
\hline Parameters & Values \\
\hline Number of frames per sample & 80 \\
\hline Frame size & $224 \times 224 \times 3$ \\
\hline Learning rate & 0.1 \\
\hline Batch Size (1 unit) & 64 \\
\hline Hidden Layers & 16 \\
\hline Epoch & 30 \\
\hline
\end{tabular}

Proposed Convolutional Deep Neural Network: Deep learning is a virtual machine that imitates the neuron network in the brain. It is a subset of machine learning and, since it makes use of deep neural networks, it is called deep learning. Layers are named as input layers, hidden layers and output layers. The deep learning means that neurons in more than two layers are connected by the network. 
The neuron must process and then transmit the input signal that the layer above it receives. In the next layer, the intensity of the signal provided to the neuron depends on the weight, bias, and role of activation. The network collects and operates vast volumes of input data over many layers; at each layer, the network can learn increasingly complex characteristics of the data. Deep learning is an efficient instrument for predicting an actionable outcome. Deep learning excels in pattern discovery (unsupervised learning) and knowledge-based prediction. Large number of data potentially helps to improve the learning. and its efficiency.

Deep Learning Process: In several functions, from object detection and other synthesis model recognition, a deep neural network offers state-of-the-art precision. Deep model will learn based on previous decision and current training model. Infants are trained based on number of instances and learning, based on physical aspects infants are getting learning. DecisionLearning works based on tail,nose and color and its learned from hierarchy complicated features.Just the same runs a network of neurons. Each layer represents a deeper level of knowledge, i.e., the hierarchy of knowledge. More complex features than with two layers can be learned by a four-layer neural network.

The learning occurs in two phases.

- Stage 1: The application of a nonlinear input transformation and the development of a statistical model as an output.

- Stage 2: Aims to develop the model with a derivative-like mathematical procedure.

These two steps are replicated hundreds to thousands of times by the neural network until it has achieved a tolerable degree of accuracy. This two-phase repeat is called an iteration.

Simulation Setup: In this research article, the proposed reliefF-KNN model is simulated by using MATLAB (2019a) environment with windows 10 operating system, 8GB RAM, Intel i5processor, and 4 TB hard disk.

\section{RESULTS AND DISCUSSION}

MobiAct is version 2 of the public dataset (FAQ. Keras Documentation. Available online: https://keras. io/ (accessed on 10 February 2020), which uses a smartphone in its pocket to collect data. Different types of activities are requested by the participants. Synthetic Data preprocessing involves three steps namely data collection, data preprocessing and data augmentation. Various datasets were collected and one example among the collected data set can be found below.

Comparison with state of the art: The performance compared with other techniques on the same Mobi Act and synthetic Human Actions dataset is shown in Table 2 and Figure 9. In reality, our strategy, based on hybrid deep learning, achieved an average of 93\%. Overall, our approach provides comparable results with the best associated work on the MobiAct dataset using deep learning for the identification of human behavior.

Figure 5: Action Prediction (Sitting)

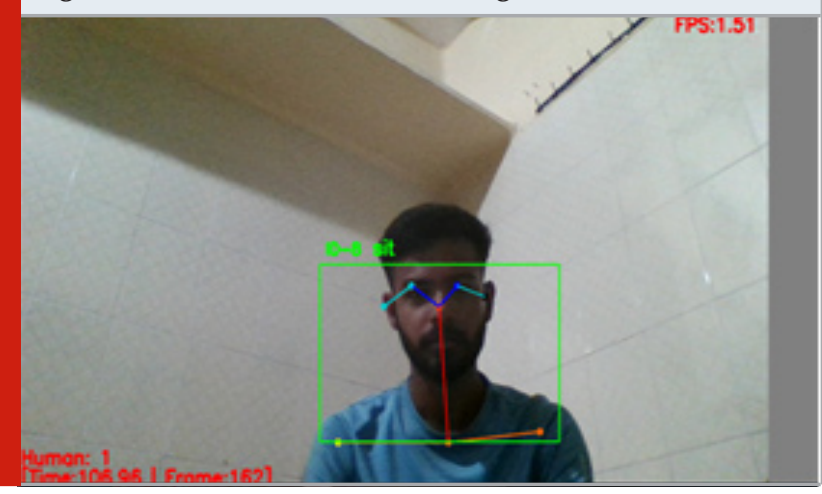

Figure 6: Action Prediction (Standing)

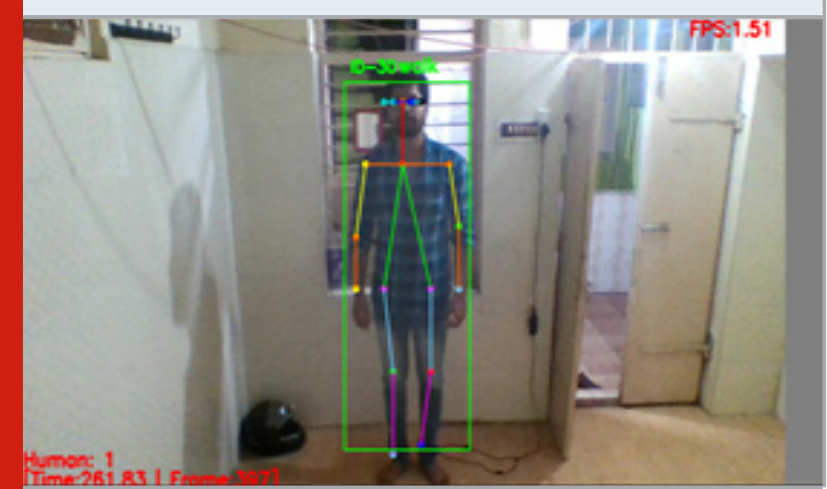

Figure 7: Action Prediction (Fall Down)

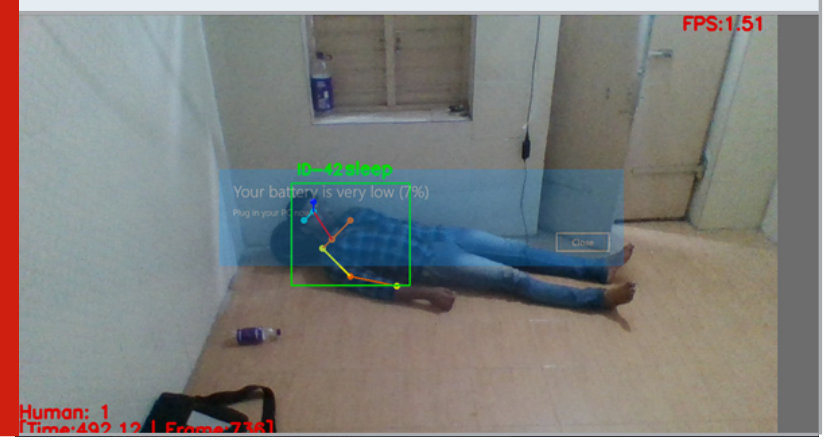

Figure 8: Human Action detection of falling down

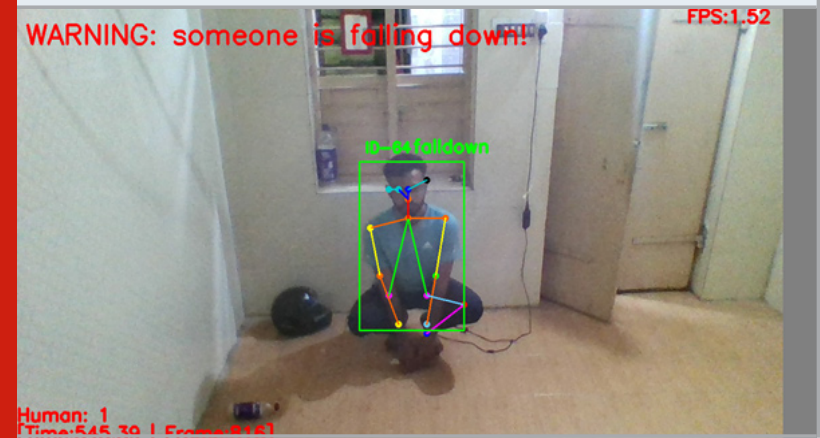


Table 2. Comparitive Analysis

\begin{tabular}{|l|l|c|c|c|c|c|}
\hline Data set & Metrics & LSTM [16] & CNN [16] & $\begin{array}{c}\text { CNN- } \\
\text { LSTM [16] }\end{array}$ & $\begin{array}{c}\text { Auto Encoder - } \\
\text { CNN -LSTM [16] }\end{array}$ & Proposed \\
\hline MobiAct & Accuray & $93 \%$ & $91 \%$ & $93 \%$ & $98 \%$ & $99.3 \%$ \\
\hline Synthetic & Accuray & $92 \%$ & $89 \%$ & $91 \%$ & $95 \%$ & $96.3 \%$ \\
\hline
\end{tabular}

Figure 9: Comparitive Analysis

Accuracy Comparision
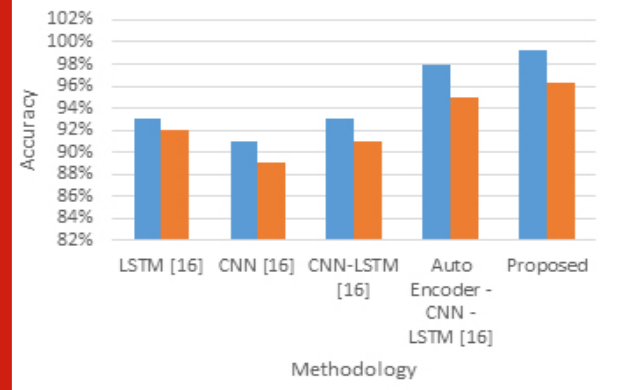

MobiAct Accuray nsynthetic Accuray

Methodology

The proposed model achieved a 98 percent accuracy rate and (Meriem \&t Chikhaoui 2020) exploited the efficiency of the Support Vector Machine fully convolutional neural network model to predict human behavior.

\section{CONCLUSION}

This project is a system of recognition of human behavior and recognition of human movements that can automatically identify human everyday activities using the latest deep learning approach. We develop an effective skeleton information based HAR. It will recognize the human activities effectively. We have developed a react native application so that we can view the live streaming with a notification when an abnormal activity is detected. So, we can save our grandparents from sudden health issues and can also help the old age homes for taking care of the elder people. In the coming future, we review the application of the human activity sensing technology in the recognition field and it can promote for all type of recognition with more accuracy compared to this project. In this field there are more chance to develop or convert this project in many ways. The accuracy of the prediction will be increased by using different efficient techniques and algorithms. Avoids mishaps with elderly as well as children.

\section{REFERENCES}

Alfredo Nazabal, ' Student Member, IEEE, Pablo GarcuaMoreno, Antonio Artes-Rodruguez, Senior Member, IEEE, Zoubin Ghahramani. "Human Activity Recognition by Combining a Small Number of Classifiers” [2017, Vol No 2716-6112]

Anshul Mittal, Pradeep Kumar, Partha Pratim Roy, Raman Balasubramanian And Bidyut B. Chaudhuri “A Modified-Lstm Model For Continuous Sign Language
Recognition Using Leap Motion” [2019, Vol No15581748 ]

Chhavi Dhiman, Member IEEE, Dinesh Kumar Vishwakarma, Senior Member, IEEE "View-invariant Deep Architecture for Human Action Recognition using Two-stream Motion and Shape Temporal Dynamics”[2020, Vol No:0098-3063]

Cho Nilar Phyo , Student Member, Ieee, Thi Thi Zin, Member, Ieee And Pyke Tin “Deep Learning For Recognizing Human Activities Using Motions of Skeletal Joints” [2018,Vol No: 0098-3063]

Dapeng Tao, Lianwen Jin, Member, Ieee, Yuan Yuan, Senior Member, Ieee, And Yang Xue "Ensemble Manifold Rank Preserving For Acceleration-Based Human Activity Recognition” [2016, Vol No: 2162237X]

FAQ. Keras Documentation. Available online: https:// keras.io/ (accessed on 10 February 2020).

Hao Zhang, Member, Ieee, Wenjun Zhou, Member, Ieee, And Lynne E. Parker, Fellow, Ieee "Fuzzy Temporal Segmentation And Probabilistic Recognition of Continuous Human Daily Activities" [2015, Vol No: 2168-2291]

Jianjie Lu And Kai-Yu Tong, Senior Member, Ieee "Robust Single Accelerometer-Based Activity Recognition Using Modified Recurrence Plot” [2019,Vol No: 1558-1748] Jin Qi , Zhangjing Wang, Xiancheng Lin, And Chunming Li "Learning Complex Spatio-Temporal Configurations Of Body Joints For Online Activity Recognition” [2018, Vol No: 2168-2291]

Lei Wang, Student Member, IEEE, Xu Zhao*, Member, IEEE, Yunfei Si, Liangliang Cao, Member, IEEE, and Yuncai Liu, Member, IEEE "Context-Associative Hierarchical Memory Model For Human Activity Recognition And Prediction” [2016, Vol No: 15209210]

P.V.V. Kishore, Senior Member, Ieee, D.Anil Kumar, Student, Ieee, A.S.C.S.Sastry, Member, Ieee, And E.Kiran Kumar, Student, Ieee. "Motionlets Matching With Adaptive Kernels For 3D Indian Sign Language Recognition" [2018, Vol No: 1558-1748]

Siyuan Qi, Baoxiong Jia, Siyuan Huang, Ping Wei, and Song-Chun Zhu "A Generalized Earley Parser for Human Activity Parsing and Prediction"[2020, Vol No: 
0162-8828]

Sofia Savvaki, Grigorios Tsagkatakis , Athanasia Panousopoulou , and Panagiotis Tsakalides "Matrix and Tensor Completion on a Human Activity Recognition Framework" [2017, Vol No 2716-6112]

Wanru Xu, Zhenjiang Miao, Member, Ieee, Xiao-Ping Zhang, Senior Member, Ieee, Yi Tian "A Hierarchical Spatio-Temporal Model For Human Activity Recognition” [2017,Vol No: 1520-9210]

Zerkouk, Meriem, and Belkacem Chikhaoui. "Spatiotemporal abnormal behavior prediction in elderly persons using deep learning models." Sensors 20.8 (2020): 2359.

Zhelong Wang, Donghui Wu, Jianming Chen, Ahmed Ghoneim and M. Anwar Hossain "A Triaxial Accelerometer-Based Human Activity Recognition via EEMD-Based Features and Game-Theory-Based Feature Selection"[2015, Vol No :1530-437]

Zhelong Wang, Donghui Wu, Jianming Chen, Ahmed Ghoneim and M. Anwar Hossain "A Triaxial Accelerometer-Based Human Activity Recognition via EEMD-Based Features and Game-Theory-Based Feature Selection” [2015, Vol No 1530-437] 